Clinical Case Report

\title{
The Importance of Early Recognition and Prompt Treatment of Adrenal Crisis and Addison's Disease
}

\author{
Ivona Butorac Ahel ${ }^{1}$, Srećko Severinski ${ }^{1}$, Kristina Lah Tomulić ${ }^{1}$, Arijan Verbić ${ }^{1}$, Ana Milardović ${ }^{1}$, \\ Mladen Jašić ${ }^{2}$
}

${ }^{1}$ Department of Pediatrics, Clinical Hospital Center Rijeka, Croatia, ${ }^{2}$ Department of Pediatrics, General Hospital Pula, Croatia

Correspondence: ivonabuah@gmail.com Tel.: + 38551659157

Fax.: + 38551623126

Received: Mart 2, 2019

Accepted: May 5, 2019

Key Words: Adrenal Crisis - Adrenal Insufficiency - Emergency.
Objective - The aim of the paper was to emphasize the importance of early recognition and urgent treatment of adrenal crisis due to Addison's disease in order to prevent potential lethal outcome. Case Report - A 13-year-old boy was presented to the emergency room with 2-days of vomiting, fatigue, and weakness, without diarrhea or fever. On admission he was in hypovolemic shock, febrile and hypoglycemic. The initial diagnosis was septic shock and the patient was treated with intravenous boluses, dobutamine and antibiotics. Blood pressure was consistently low despite adequate dose of dobutamine and intravenous hydration and the patient had hypoglycemia on two occasions. Subsequently, Addison's disease was diagnosed on the basis of hyperpigmentation of the skin, low basal cortisol and high ACTH. He rapidly responded to intravenous stress doses of hydrocortisone, followed by hydrocortisone and fludrocortisone oral replacement therapy. Conclusion - Addison's disease can be easily missed due to nonspecific symptoms and presentations, rarity of the condition, and low index of suspicion. In every patient with hyperpigmentation, hyponatremia, hyperkalemia, acidosis, and hypoglycemia, the diagnosis of Addison's disease should be strongly suspected.

\section{Introduction}

Primary adrenal insufficiency (PAI) is characterized by deficient biosynthesis of glucocorticoids, with or without deficiency in mineralocorticoids and adrenal androgens. PAI was first described by Thomas Addison and is therefore commonly termed Addison's disease $(\mathrm{AD})(1)$. It is rare in children and adults with the incidence of 0.8 per $100000 /$ year $(2,3)$. The most common cause of PAI in newborns and infants is congenital adrenal hyperplasia $(\mathrm{CAH})$ but in adolescents the most common cause is Addison's disease caused by autoimmune destruction of adrenal glands (4). Other causes of PAI include inherited developmental and metabolic disorders, infections, and adrenal haemorrhage. Autoimmune destruction of the adrenal gland is responsible for only $15 \%$ of PAI in children (5). Since autoimmune adrenalitis is responsible for over $80 \%$ of adrenal insufficiency in adults, the term $\mathrm{AD}$ is now widely used to indicate autoimmune etiology of the disease (2). PAI may have an acute or chronic presentation. In many cases acute presentation reflect an undiagnosed underlying chronic or developmental process. Acute adrenal insufficiency known as adrenal crisis or Addisonian crisis is a life-threatening medical emergency which requires prompt diagnosis 
and treatment. It may be the first presentation in up to $50 \%$ of patients with adrenal insufficiency (2).

We report a case of adrenal crisis caused by $\mathrm{AD}$ to emphasize the importance of early recognition and prompt treatment in order to prevent potential lethal outcome.

\section{Case Report}

A previously healthy 13-year-old boy, a tourist from Germany, presented to the emergency department with a two-days history of vomiting, weakness and fatigue. Until arrival to the emergency department he has been vomited approximately 13 times in two days and had no diarrhea or fever. At presentation, the patient looked ill and in distress. Clinical examination revealed severe dehidration with signs of hypovolemic shock, heart rate of 140 beats $/ \mathrm{min}$, a blood pressure of $60 / 40 \mathrm{~mm} \mathrm{Hg}$, prolonged capillary refilled time ( 4 seconds) and cyanotic and cold pe- riphery. He had fever $\left(38.2^{\circ} \mathrm{C}\right)$ and blood glucose level was $2.2 \mathrm{mmol} / \mathrm{L}$. The boy immediately received two $0.9 \%$ sodium chloride $20 \mathrm{ml} / \mathrm{kg}$ fluid boluses and hypoglycemia was corrected using $10 \%$ dextrose $3 \mathrm{ml} /$ $\mathrm{kg}$ bolus. This was followed by intravenous $5 \%$ dextrose in $0.9 \%$ sodium chloride fluid infusion, and was transferred to the intensive care unit. Laboratory investigations showed severe metabolic acidosis, renal dysfunction, mild hyponatremia and hyperkalemia (Table 1). Since the patient had signs of shock in association with fever, leucocytosis, neutrophilia, and raised CRP, our working diagnosis was septic shock. Inotropic support with dobutamine and antibiotic therapy with ceftriaxone was started after taking blood culture. The patient remained hypotensive and tachycardic despite adequate fluid resuscitation and appropriate dose of dobutamine were administered. He was hypoglycemic at two occasions $(2.2 \mathrm{mmol} / \mathrm{L}$ and $2.0 \mathrm{mmol} / \mathrm{L}$ respectively). The next morning generalized

\section{Table 1. Results of Laboratory Investigations}

\begin{tabular}{llll}
\hline Laboratory tests (normal range) & On admission & 8 hours after admission & 12 hours after admission \\
\hline Erythrocytes $\left(4.43-5.88 \times 10^{12} / \mathrm{L}\right)$ & 5.08 & 4.32 & - \\
\hline Hemoglobin $(129-166 \mathrm{~g} / \mathrm{L})$ & 138 & 114 & - \\
\hline Leukocytes $\left(4.4-11.6 \times 10^{9} / \mathrm{L}\right)$ & 15.7 & 8.9 & - \\
\hline C-reactive protein $(0.1-2.8 \mathrm{mg} / \mathrm{L})$ & 69 & 124.5 & - \\
\hline Sodium $(135-145 \mathrm{mmol} / \mathrm{L})$ & 131 & 132 & - \\
\hline Potassium $(3.7-5.0 \mathrm{mmol} / \mathrm{L})$ & 5.3 & 4.6 & - \\
\hline Chloride $(95-106 \mathrm{mmol} / \mathrm{L})$ & 96 & 104 & - \\
\hline Blood urea nitrogen $(2.7-6.8 \mathrm{mmol} / \mathrm{L})$ & 10 & 9 & - \\
\hline Creatinine $(40-72 \mu \mathrm{mol} / \mathrm{L})$ & 144 & 72 & - \\
\hline Glucose $(3.9-5.9 \mathrm{mmol} / \mathrm{L})$ & 3.3 & 4.7 & - \\
\hline pH $(7.35-7.35)$ & 7.09 & 7.32 & - \\
\hline HCO3 $(18-23 \mathrm{mmol} / \mathrm{L})$ & 8.2 & 13.6 & 17.19 \\
\hline BE $(-4-2 \mathrm{mmol} / \mathrm{L})$ & -20.2 & -12 & 364.6 \\
\hline Cortisol $(171-536 \mathrm{nmol} / \mathrm{L})$ & - & - & 12.6 \\
\hline ACTH $(1.6-13.9 \mathrm{pmol} / \mathrm{L})$ & - & - & 147.78 \\
\hline Aldosterone $(28-144 \mathrm{ng} / \mathrm{L})$ & - & - & - \\
\hline Renin $(3.18-32.61 \mathrm{pg} / \mathrm{ml})$ & - & - & - \\
\hline
\end{tabular}


hyperpigmentation was noticed, especially in mucous membranes, pressure points, and palmar creases (Fig. 1).

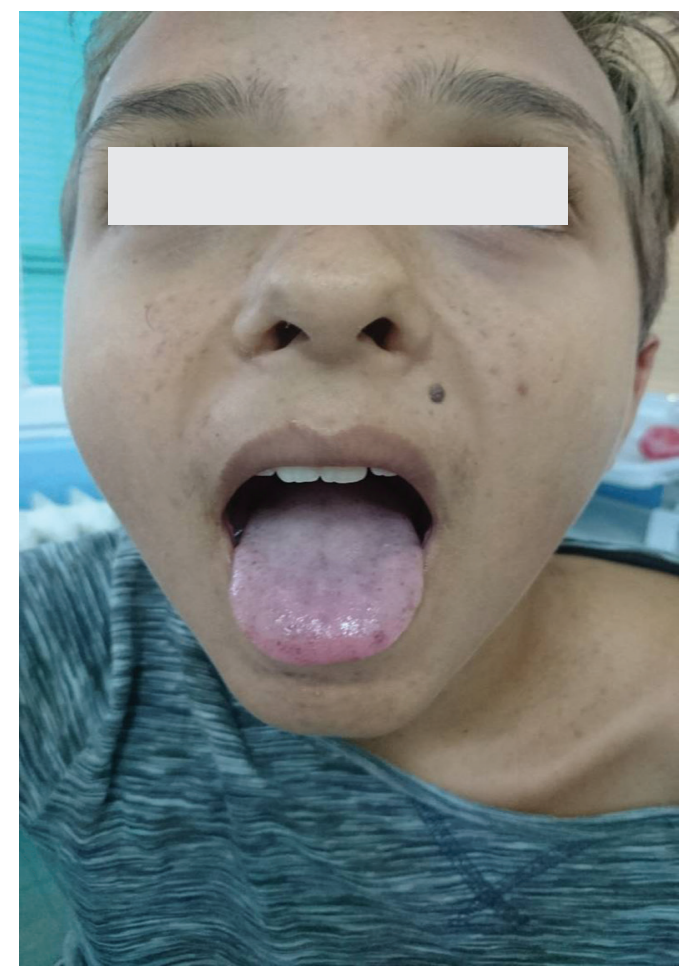

Fig. 1. Hyperpigmentation of the Tongue and Face.

Based on clinical presentation and blood chemistry, adrenal crisis was suspected and the treatment with $100 \mathrm{mg}$ of hydrocortisone 4 hourly was immediately started. Baseline low plasma cortisol level and high ACTH level confirmed the supposed diagnosis of Addisonian crisis (Table 1). After the first dose of hydrocortisone the patient was feeling much better and he became normotensive. The symptoms were completely withdrawn after 36 hours and intravenous therapy was switched to triple dose oral hydrocortisone therapy which was gradually reduced to maintenance level $(10 \mathrm{mg} / \mathrm{m} 2 /$ day). Further investigations revealed low aldosterone and renin level and oral fludrocortisone was initiated (Table 1). We found out that the boy felt tired and had dark skin for about a year. Education on adrenal insufficiency and stress dose of hydrocortisone were provided to the boy and his parents. He was discharged after 7 days in a good clinical condition with the recommendation to contact the doctor immediately in his home country. We had no possibility to investigate the adrenal antibodies.

\section{Discussion}

Adrenal crisis should be considered in every patient presenting with one or more of the following simptoms: circulatory collapse, altered consciousness, hypoglycaemia, hyponatremia, hyperkalemia, seizures, history of steroid use/withdrawal, or any clinical features of AD. Patients presenting with adrenal crisis are often in hypotensive shock which occurs secondary to hypovolemia, but also due to hypocortisolism, as glucocorticoids exert a permissive effect on catecholamine action. If not recognized, it may be refractory to fluids and inotropes such as adrenaline and dobutamine (6). Hyponatremia is due to concomitant aldosterone deficiency, which leads to natriuresis, volume depletion, and hyperkalemia (6). Hypoglycaemia occurs because of loss of the gluconeogenic effects of cortisol. Hyperpigmentation is the one of the commonest and most striking feature of $\mathrm{AD}$ and it is due to excessive ACTH stimulation of the melanocortin 1 receptor (MC1R). Common sites involve the extensor surfaces, axillae, nipples, old scars, palmar creases, pressure points and mucous membranes but may also become generalized.

Except for salt craving, the symptoms of $\mathrm{AD}$ are rather nonspecific and include weakness, fatigue, musculoskeletal pain, weight loss, abdominal pain, memory impairment, depression, and anxiety. As a consequence, the diagnosis is frequently delayed, resulting in a clinical presentation with an acute life-threatening adrenal crisis (7). In more 
than $90 \%$ of cases of adrenal crisis, there is a known precipitating event (6). The most common precipitanting factor is gastrointestinal illness. Since it is a medical emergency, in patient who is clinically unstable, the treatment should not be delayed for the purpose of diagnostic tests. Serum cortisol, ACTH, aldosterone, renin and dehydroepiandrosterone-sulfate can be taken just before hydrocortisone administration and may be useful in the diagnosis of adrenal insufficiency. A low serum cortisol level of $<3 \mathrm{mcg} / \mathrm{dL}$ ( 80 $\mathrm{nmol} / \mathrm{L}$ ) done in the early morning or in a state of stress, in the presence of markedly elevated ACTH level confirms the diagnosis of $\mathrm{AD}(8)$.

Since signs and symptoms of $\mathrm{AD}$ are nonspecific, serum electrolytes abnormalities often provide a clue to diagnosis. Hyponatremia is the most common abnormality seen in $\mathrm{AD}$. The differential diagnosis for hyponatremia in children is broad and can be divided into hypovolemic, normovolemic and hypervolemic hyponatremia. Hypovolemic hyponatremia can be caused by gastroenteritis, renal salt wasting and intense exercise. Conditions associated with renal salt wasting include cerebral salt wasting, primary renal tubular disorders and adrenal insufficiency, including $\mathrm{AD}, \mathrm{CAH}$ and hypoaldosteronism. Normovolemic causes include syndrome of inappropriate antidiuretic hormone (SIADH) and primary polydipsia. Hypervolemic causes include nephrotic syndrome, renal failure and heart failure (8). Positive adrenal autoantibodies establish the diagnosis of $\mathrm{AD}$ which can be a part of autoimmune poliglandular syndrome or it may be an isolated disorder. In all males diagnosed with PAI without evidence of autoimmunity, X-linked adrenoleukodystrophy should be rulled out.

The first-line management is based on fluid resuscitation and stress-dosing glucocorticoid replacement. Hypovolemia and hypona- tremia are treated by rapid intravenous fluid boluses with isotonic sodium chloride $0.9 \%$. Hypoglycemia is corrected with intravenous $5 \%$ dextrose. As for glucocorticoid replacement, parenteral hydrocortisone should be administered immediately to prevent further clinical deterioration. Stress doses of glucocorticoids also provide mineralocorticoid activity. (9). Awareness of this life-threatening condition and its inclusion in the differential diagnosis of unexplained shock and prompt treatment is life safing.

Since cortisol is stress hormone, surgery, anesthesia, trauma, and illnesses result in increased plasma ACTH and cortisol levels. In order to prevent the future risks of adrenal crisis, all patients with $\mathrm{AD}$ sholud be educated about stress-related glucocorticoid dose adjustment. During conditions of moderate stress, the usual glucocorticoid replacement dose should be doubled. In major stress the hydrocortisone dose should be increased to three to four times the normal replacement. If the patient is unable to tolerate oral therapy, hydrocortisone should be administered by parenteral route.

In our patient $\mathrm{AD}$ probably began more than one year ago and adrenal crisis was provoked by stomac flu. The presence of hyperpigmentation, hypotension along with electrolyte abnormalities of hyponatremia and hyperkalemia led to the suspicion of AD. Since he was admitted in the middle of the night the hyperpigmentation wasn't noticed and the signs of shock and high inflammatory parameters mislead us to the diagnosis of septic shock. Fortunately, the right diagnosis was made after a few hours and possible catastrophic consequences were prevented.

\section{Conclusion}

Since $\mathrm{AD}$ is rare disease, it can remain unrecognized or can easily be misdiagnosed if we don't think of it. Because of that, in every pa- 
tient with unexplained circulatory collapse, hypotension, vomiting, or diarrhea, the diagnosis of $\mathrm{AD}$ should be considered. In patients with hyperpigmentation, hyponatremia, hyperkalemia, acidosis, and hypoglycemia, the diagnosis should be strongly suspected.

Authors' Contributions: Conception and design: IBA, KLT and MJ; Drafting the article: IBA; Revising it critically for important intellectual content: IBA, SS, KLT, AM, AV and MJ; Approved final version of the manuscript: IBA, SS, KLT, AM, AV and MJ.

Conflict of Interest: The authors declare that they have no conflict of interest.

\section{References}

1. Bishop PM. The history of the discovery of Addison's disease. Proc R Soc Med. 1950;43(1):35-42.

2. O’Connell S, Siafarikas A. Addison disease - diagnosis and initial management. Aust Fam Physician. 2010;39(11):834-7.

3. Flück CE. MECHANISMS IN ENDOCRINOLOGY: Update on pathogenesis of primary adrenal insufficiency: beyond steroid enzyme deficiency and autoimmune adrenal destruction. Eur J Endocrinol. 2017;177(3):R99-R111.

4. Porter J, Blair J, Ross RJ. Is physiological glucocorticoid replacement important in children? Arch Dis Child. 2017;102:199-205.

5. Perry R, Kecha O, Paquette, Huot C, Van Vliet G, Deal C. Primary adrenal insufficiency in children: twenty years experience at the Sainte-Justine Hospital, Montreal. J Clin Endocrinol Metab. 2005;90:3243-50

6. Puar TH, Stikkelbroeck NM, Smans LC, Zelissen PM, Hermus AR. Adrenal Crisis: Still a Deadly Event in the 21st Century. Am J Med. 2016;129(3):339.e1-9.

7. Bleicken B, Hahner S, Ventz M, Quinkler M. Delayed diagnosis of adrenal insufficiency is common: a cross-sectional study in 216 patients. Am J Med Sci. 2010;339:525-31.

8. Bowden SA, Henry R. Pediatric Adrenal Insufficiency: Diagnosis, Management, and New Therapies. Int J Pediatr. 2018 Nov 1;2018:1739831. Epub 2018 Nov 1.

9. Bornstein SR, Allolio B, Arlt W, Barthel A, DonWauchope A, Hammer GD, et al. Diagnosis and treatment of primary adrenal insufficiency: an Endocrine Society clinical practice guideline. J Clin Endocrinol Metab. 2016;101:364-89. 